\title{
A light-front coupled-cluster method for quantum field theories
}

\author{
John HILLER ${ }^{* \dagger}$ \\ University of Minnesota-Duluth, USA \\ E-mail: jhillered.umn.edu
}

\begin{abstract}
The Hamiltonian eigenvalue problem for bound states of a quantum field theory is formulated in terms of Dirac's light-front coordinates and then approximated by the exponential-operator technique of the standard coupled-cluster method. This approximation eliminates any need for the usual approximation of Fock-space truncation. Instead, the exponential operator is truncated and the terms retained are determined by a set of nonlinear integral equations. These equations are solved simultaneously with an effective eigenvalue problem in the valence sector, where the number of constituents is small. Matrix elements can be calculated, with extensions of techniques from standard coupled-cluster theory.
\end{abstract}

Sixth International Conference on Quarks and Nuclear Physics

April 16-20, 2012

Ecole Polytechnique, Palaiseau, Paris

\footnotetext{
* Speaker.

${ }^{\dagger}$ This work was done in collaboration with S.S. Chabysheva and supported in part by the US Department of Energy and the Minnesota Supercomputing Institute.
} 


\section{Introduction}

The light-front coupled-cluster (LFCC) method [1] is intended as a method for the calculation of hadron structure in terms of Fock-state wave functions without the usual truncation of Fock space. The Fock-state representation of a hadron, such as a proton, takes the form

$$
|p\rangle=\psi_{\text {und }}|u u d\rangle+\psi_{\text {und }}|u u d g\rangle+\psi_{\text {uudgg }}|u u d g g\rangle+\psi_{\text {uudq } \bar{q}}|u u d q \bar{q}\rangle+\cdots .
$$

This is to be an eigenstate of the QCD Hamiltonian

$$
\left(\text { K.E. }+V_{\mathrm{QCD}}\right)|p\rangle=E_{p}|p\rangle
$$

with $E_{p}=\sqrt{m_{p}^{2}+p^{2}}$ and $V_{\mathrm{QCD}}$ the interaction terms of gluon emission and absorption by quarks, quark-antiquark production and annihilation, and the three and four-gluon vertices. This is equivalent to a set of coupled integral equations for the wave functions.

Usually, this infinite set of equations is made finite by truncation of Fock space; however, this introduces uncanceled divergences [2]. For example, the Ward identity of gauge theories is destroyed by truncation, because the limitation on the number of particles allowed in intermediate states removes some of the contributions to the identity. There is a direct analog in Feynman perturbation theory, where separation of a covariant diagram into time-ordered diagrams, and removal of those that include intermediate states with more particles than some finite limit, destroys covariance, disrupts regularization, and induces spectator dependence for subdiagrams. In the nonperturbative case, this happens not just to some finite order in the coupling but to all orders.

The method is formulated in terms of light-cone coordinates [3,4]. The time coordinate is $x^{+}=t+z$, and the spatial coordinates are $\underline{x}=\left(x^{-}, \vec{x}_{\perp}\right)$, with $x^{-} \equiv t-z$ and $\vec{x}_{\perp}=(x, y)$. The light-cone energy is $p^{-}=E-p_{z}$, and the momentum is $\underline{p}=\left(p^{+}, \vec{p}_{\perp}\right)$, with $p^{+} \equiv E+p_{z}$ and $\vec{p}_{\perp}=\left(p_{x}, p_{y}\right)$. This leaves the mass-shell condition $p^{2}=m^{2}$ as $p^{-}=\frac{m^{2}+p_{\perp}^{2}}{p^{+}}$and the mass eigenvalue problem as [5]

$$
\mathscr{P}^{-}|\underline{P}\rangle=\frac{M^{2}+P_{\perp}^{2}}{P^{+}}|\underline{P}\rangle, \quad \underline{\mathscr{P}}|\underline{P}\rangle=\underline{P}|\underline{P}\rangle .
$$

The advantages of this coordinate choice include the absence of spurious vacuum contributions to eigenstates and a boost-invariant separation of internal and external momenta. Vacuum contributions are suppressed because $p^{+}$is positive for all particles; the vacuum cannot produce particles and conserve $p^{+}$. The separation of internal momenta is obtained by defining longitudinal momentum fractions $x_{i} \equiv p_{i}^{+} / P^{+}$and relative transverse momenta $\vec{k}_{i \perp} \equiv \vec{p}_{i \perp}-x_{i} \vec{P}_{\perp}$.

The original coupled-cluster (CC) method was developed for the many-body Schrödinger equation in nuclear physics [6] and later applied to the many-electron problem in molecules [7]. The method has become an important tool in nuclear physics and physical chemistry for the $\mathrm{N}$ body problem in nonrelativistic quantum systems [8]. The basic idea is to form an eigenstate as $e^{T}|\phi\rangle$, where $|\phi\rangle$ is a product of single-particle states and the terms in $T$ annihilate states in $|\phi\rangle$ and create excited states, to build in correlations; however, the number of particles does not change. The approximation made is to truncate $T$ at some number of excitations. The LFCC method uses the mathematics of these constructions but applies it to a situation where $|\phi\rangle$ contains a small number of particles, $T$ adds additional particles, and the states are eigenstates of momentum with Dirac-delta normalization. 


\section{Light-front coupled-cluster method}

To solve the fundamental eigenvalue problem $\mathscr{P}^{-}|\psi\rangle=\frac{M^{2}+P_{\perp}^{2}}{P^{+}}|\psi\rangle$ by the LFCC method [1], we write the eigenstate as $|\psi(\underline{P})\rangle=\sqrt{Z} e^{T}|\phi(\underline{P})\rangle$ and seek solutions for the valence state $|\phi(\underline{P})\rangle$ and the operator $T$. This operator contains terms that only increase particle number, while conserving $J_{z}$, light-front momentum $\underline{P}$, charge, and the other quantum numbers of the eigenstate. The constant $Z$ controls normalization, which is chosen to be $\left\langle\psi\left(\underline{P}^{\prime}\right) \mid \psi(\underline{P})\right\rangle=\delta\left(\underline{P}^{\prime}-\underline{P}\right)$, with the valence state normalized in the same way: $\left\langle\phi\left(\underline{P}^{\prime}\right) \mid \phi(\underline{P})\right\rangle=\delta\left(\underline{P}^{\prime}-\underline{P}\right)$. Because $p^{+}$is positive, $T$ must include annihilation, and powers of $T$ include contractions. This converts the original eigenvalue problem into an eigenvalue problem for the valence state

$$
P_{v} \overline{\mathscr{P}^{-}}|\phi(\underline{P})\rangle=\frac{M^{2}+P_{\perp}^{2}}{P^{+}}|\phi(\underline{P})\rangle,
$$

with $\overline{\mathscr{P}}-=e^{-T} \mathscr{P}^{-} e^{T}$ the effective Hamiltonian and $P_{v}$ the projection onto the valence Fock sector, and into an auxiliary equation for $T$

$$
\left(1-P_{v}\right) \overline{\mathscr{P}^{-}}|\phi(\underline{P})\rangle=0 .
$$

Calculation of expectation values, and more generally matrix elements, requires some care, to avoid any necessity of computing the infinite sum implied by the inner product $\left\langle\phi\left|e^{T^{\dagger}} e^{T}\right| \phi\right\rangle$. This can be done, with use of constructions from the CC method [8]. The expectation value

$$
\langle\hat{O}\rangle=\frac{\left\langle\phi\left|e^{T^{\dagger}} \hat{O} e^{T}\right| \phi\right\rangle}{\left\langle\phi\left|e^{T^{\dagger}} e^{T}\right| \phi\right\rangle}
$$

for an operator $\hat{O}$ is rewritten as $\langle\hat{O}\rangle=\langle\tilde{\psi}|\bar{O}| \phi\rangle$ in terms of an effective operator $\bar{O} \equiv e^{-T} \hat{O} e^{T}$ and a left eigenvector of $\overline{\mathscr{P}}$ :

$$
\langle\tilde{\psi}|=\langle\phi| \frac{e^{T^{\dagger}} e^{T}}{\left\langle\phi\left|e^{T^{\dagger}} e^{T}\right| \phi\right\rangle} .
$$

The effective operator can be computed from the Baker-Hausdorff expansion $\bar{O}=\hat{O}+[\hat{O}, T]+$ $\frac{1}{2}[[\hat{O}, T], T]+\cdots$. The bra $\langle\tilde{\psi}|$ is seen to be a left eigenstate by the following steps:

$$
\langle\tilde{\psi}| \overline{\mathscr{P}-}=\langle\phi| \frac{e^{T^{\dagger}} \mathscr{P}^{-} e^{T}}{\left\langle\phi\left|e^{T^{\dagger}} e^{T}\right| \phi\right\rangle}=\langle\phi| \overline{\mathscr{P}^{-}} \frac{e^{T^{\dagger}} e^{T}}{\left\langle\phi\left|e^{T^{\dagger}} e^{T}\right| \phi\right\rangle}=\frac{M^{2}+P_{\perp}^{2}}{P^{+}}\langle\tilde{\psi}| .
$$

Also, it has the normalization

$$
\left\langle\tilde{\psi}\left(\underline{P}^{\prime}\right) \mid \phi(\underline{P})\right\rangle=\left\langle\phi\left(\underline{P}^{\prime}\right)\left|\frac{e^{T^{\dagger}} e^{T}}{\left\langle\phi\left|e^{T^{\dagger}} e^{T}\right| \phi\right\rangle}\right| \phi(\underline{P})\right\rangle=\delta\left(\underline{P}^{\prime}-\underline{P}\right) .
$$

As formulated to this point, the new valence eigenvalue problem and the auxiliary condition for $T$ provide an exact representation of the original eigenvalue problem. The LFCC method then invokes a truncation, not of Fock space but of the terms in $T$ and in the projection $1-P_{v}$. This leads to a finite set of auxiliary equations for a finite set of functions that define the truncated $T$ operator and to a finite number of terms in the valence projection of the effective Hamiltonian $\overline{\mathscr{P}}$. The latter is conveniently expressed in terms of a Baker-Hausdorff expansion where the number of contributing terms is finite. Similarly, the contributions to matrix elements of operators are also finite in number. 


\section{Application to a soluble model}

To illustrate the method, we apply it [1] to an exactly soluble model [9], a light-front analog of the Greenberg-Schweber model with a static fermionic source that emits and absorbs bosons without changing its spin. The model lacks full covariance because of the static source; all states are limited to having a fixed total transverse momentum $\vec{P}_{\perp}$, which we take to be zero. In this context, not all features of the method are apparent, but the model is sufficient to show how the method is used.

The light-front Hamiltonian is [1]

$$
\begin{aligned}
& \mathscr{P}^{-}=\int d \underline{p} \frac{M^{2}+M_{0}^{\prime} p^{+}}{P^{+}} \sum_{s} b_{s}^{\dagger}(\underline{p}) b_{s}(\underline{p})+\int d \underline{q} \sum_{l}(-1)^{l} \frac{\mu_{l}^{2}+q_{\perp}^{2}}{q^{+}} a_{l}^{\dagger}(\underline{q}) a_{l}(\underline{q}) \\
& +\frac{g}{P^{+}} \int \frac{d \underline{p} d \underline{q}}{\sqrt{16 \pi^{3} q^{+}}} \sum_{l s}\left(\frac{p^{+}}{p^{+}+q^{+}}\right)^{\gamma}\left[a_{l}^{\dagger}(\underline{q}) b_{s}^{\dagger}(\underline{q}) b_{s}(\underline{p}+\underline{q})+b_{s}^{\dagger}(\underline{p}+\underline{q}) b_{s}(\underline{p}) a_{l}(\underline{q})\right],
\end{aligned}
$$

where $a_{0}^{\dagger}$ creates a "physical" boson of mass $\mu_{0}, a_{1}^{\dagger}$ creates a Pauli-Villars (PV) boson of mass $\mu_{1}$, and $b_{s}^{\dagger}$ creates the fermion with mass $M$ and spin $s$. The valence state is the bare-fermion state $\left|\phi^{\sigma}(\underline{P})\right\rangle=b_{\sigma}^{\dagger}(\underline{P})|0\rangle$. The $T$ operator is truncated to a single boson emission:

$$
T=\sum_{l s} \int d \underline{q} d \underline{p} t_{l s}(\underline{q}, \underline{p}) a_{l}^{\dagger}(\underline{q}) b_{s}^{\dagger}(\underline{p}) b_{s}(\underline{p}+\underline{q}),
$$

and the projection $1-P_{v}$ is truncated to the one-fermion/one-boson sector.

This form generates the exact solution, with

$$
t_{l s}(\underline{q}, \underline{p})=\frac{-g}{\sqrt{16 \pi^{3} q^{+}}}\left(\frac{p^{+}}{p^{+}+q^{+}}\right)^{\gamma} \frac{q^{+} / P^{+}}{\mu_{l}^{2}+q_{\perp}^{2}} .
$$

The fermion self-energy contribution $M_{0}^{\prime}$ is the same in all Fock sectors and the effective Hamiltonian [1] contains all three of the contributions analogous to those for the Ward identity in QED. The fact that the self-energy loop is the same in the valence sector and the one-fermion/one-boson sector plays a critical role in yielding the exact solution.

To compute an observable, we consider the Dirac form factor for the dressed fermion. It can be obtained from a matrix element of the current $J^{+}=\bar{\psi} \gamma^{+} \psi$ coupled to a photon of momentum $q$. The matrix element is generally [10]

$$
\left\langle\psi^{\sigma}(\underline{P}+\underline{q})\left|16 \pi^{3} J^{+}(0)\right| \psi^{ \pm}(\underline{P})\right\rangle=2 \delta_{\sigma \pm} F_{1}\left(q^{2}\right) \pm \frac{q^{1} \pm i q^{2}}{M} \delta_{\sigma \mp} F_{2}\left(q^{2}\right),
$$

with $F_{1}$ and $F_{2}$ the Dirac and Pauli form factors. In the model, the fermion cannot flip its spin; therefore, $F_{2}$ is zero, and we compute only $F_{1}$. Also, there are no contributions from fermionantifermion pairs, so that the current is simply

$$
J^{+}(0)=2 \sum_{s} \int \frac{d \underline{p^{\prime}}}{\sqrt{16 \pi^{3}}} \int \frac{d \underline{p}}{\sqrt{16 \pi^{3}}} b_{s}^{\dagger}\left(\underline{p^{\prime}}\right) b_{s}(\underline{p})
$$

In the LFCC method, the form factor is approximated by the matrix element

$$
F_{1}\left(q^{2}\right)=8 \pi^{3}\left\langle\widetilde{\psi}^{ \pm}(\underline{P}+\underline{q})\left|\overline{J^{+}(0)}\right| \phi^{ \pm}(\underline{P})\right\rangle,
$$


with $\overline{J^{+}(0)}=J^{+}(0)+\left[J^{+}(0), T\right]+\cdots$. The truncated left-hand eigenvector is

$$
\left\langle\widetilde{\psi}^{\sigma}(\underline{P})\right|=\left\langle\phi^{\sigma}(\underline{P})\right|+\sum_{l s} \int d \underline{q} \theta\left(P^{+}-q^{+}\right) l_{l s}^{\sigma *}(\underline{q}, \underline{P})\langle 0| a_{l}(\underline{q}) b_{s}(\underline{P}-\underline{q}),
$$

where $l_{l s}^{\sigma}$ is the left-hand one-fermion/one-boson wave function. If this wave function is assumed to take the form

$$
l_{l s}^{\sigma}(\underline{q}, \underline{P})=\delta_{\sigma s} \frac{-g}{\sqrt{16 \pi^{3} q^{+}}}\left(\frac{P^{+}-q^{+}}{P^{+}}\right)^{\gamma} \frac{q^{+} / P^{+}}{\mu_{l}^{2}+q_{\perp}^{2}} \tilde{l}\left(q^{+} / P^{+}\right),
$$

substitution into the left-hand eigenvalue problem yields a one-dimensional integral equation for $\tilde{l}(y)$

$$
\tilde{l}(y)=1+\frac{g^{2}}{16 \pi^{2}} \frac{\mu_{1}^{2}-\mu_{0}^{2}}{\mu_{0}^{2} \mu_{1}^{2}} \int_{0}^{1} d y^{\prime}\left(1-y^{\prime}\right)^{2 \gamma} y^{\prime}\left[(1-y)^{2} \tilde{l}\left(y^{\prime}(1-y)\right)-\tilde{l}\left(y^{\prime}\right)\right] .
$$

The solution of this equation can then be used to compute matrix elements.

Only the first two terms of the Baker-Hausdorff expansion of $\overline{J^{+}(0)}$ contribute to the matrix element. The first term contributes $1 / 8 \pi^{3}$ and second contributes

$$
\begin{aligned}
& \left\langle\widetilde{\psi}^{ \pm}(\underline{P}+\underline{q})\left|\left[J^{+}(0), T\right]\right| \phi^{ \pm}(\underline{P})\right\rangle=\frac{1}{8 \pi^{3}} \sum_{l}(-1)^{l} \int d \underline{q}^{\prime} \theta\left(P^{+}+q^{+}-q^{\prime+}\right) \\
& \quad \times l_{l \pm}^{ \pm}\left(\underline{q}^{\prime}, \underline{P}+\underline{q}\right)\left[\theta\left(P^{+}-q^{\prime+}\right) t_{l \pm}\left(\underline{q}^{\prime}, \underline{P}-\underline{q}^{\prime}\right)-t_{l \pm}\left(\underline{q^{\prime}}, \underline{P}+\underline{q}-\underline{q}^{\prime}\right)\right] .
\end{aligned}
$$

The form factor is then

$$
\begin{aligned}
F_{1}\left(q^{2}\right)=1+\frac{g^{2}}{16 \pi^{2}}(1+\alpha) \frac{\mu_{1}^{2}-\mu_{0}^{2}}{\mu_{0}^{2} \mu_{1}^{2}} & {\left[\int_{0}^{1 /(1+\alpha)} d y \tilde{l}(y) y(1-y)^{\gamma}[1-(1+\alpha) y]^{\gamma}\right.} \\
& \left.-\int_{0}^{1} d y \tilde{l}(y) y(1-y)^{2 \gamma}\right],
\end{aligned}
$$

with $\alpha \equiv q^{+} / P^{+}$. In the limit of $q^{2} \rightarrow 0$, we have $\alpha=0$ and $F_{1}(0)=1$, which is exactly the unit charge in the current $J^{+}=\bar{\psi} \gamma^{+} \psi$.

\section{Summary}

The advantages of the LFCC method are the absence of Fock-space truncations that cause uncanceled divergences, elimination of Fock-sector and spectator dependence of self-energy contributions, and provision for systematic improvement, through the addition of terms to the truncated operator $T$. The terms in $T$ can be organized according to the nature and number of particles annihilated and created. Applications to theories beyond the simple model considered here are in progress, with some preliminary work on the dressed-electron state in QED already completed [11]. Additional work in QED will include consideration of the dressed-photon state, extension of the dressed-electron state to include $e^{+}-e^{-}$pairs, muonium, and positronium. Application to QCD will begin with consideration of mesons in holographic QCD [12]. 


\section{References}

[1] S.S. Chabysheva and J.R. Hiller, A light-front coupled-cluster method for the nonperturbative solution of quantum field theories, Phys. Lett. B 711 (2012) 417.

[2] S.S. Chabysheva and J.R. Hiller, On the nonperturbative solution of Pauli-Villars regulated light-front QED: A comparison of the sector-dependent and standard parameterizations, Ann. Phys. 325 (2010) 2435.

[3] P.A.M. Dirac, Forms of relativistic dynamics, Rev. Mod. Phys. 21 (1949) 392.

[4] For reviews of light-cone quantization, see M. Burkardt, Light front quantization, Adv. Nucl. Phys. 23 (2002) 1; S.J. Brodsky, H.-C. Pauli, and S.S. Pinsky, Quantum chromodynamics and other field theories on the light cone, Phys. Rep. 301 (1998) 299.

[5] H.-C. Pauli and S.J. Brodsky, Solving field theory in one-space one-time dimension, Phys. Rev. D 32 (1985) 1993; Discretized light-cone quantization: solution to a field theory in one-space one-time dimension, Phys. Rev. D 32 (1985) 2001.

[6] F. Coester, Bound states of a many-particle system, Nucl. Phys. 7 (1958) 421; F. Coester and H. Kümmel, Short-range correlations in nuclear wave functions, Nucl. Phys. 17 (1960) 477.

[7] J. Čižek, On the correlation problem in atomic and molecular systems: Calculation of wave-function components in Ursell-type expansions using quantum-field theoretical methods, J. Chem. Phys. 45 (1966) 4256.

[8] For reviews of the coupled-cluster method, see H. Kümmel, K.H. Lührmann, and J.G. Zabolitzky, Many-fermion theory in expS- (or coupled cluster) form, Phys. Rep. 36 (1978) 1; R.J. Bartlett and M. Musial, Coupled-cluster theory in quantum chemistry, Rev. Mod. Phys. 79 (2007) 291.

[9] S.J. Brodsky, J.R. Hiller, and G. McCartor, Pauli-Villars as a nonperturbative ultraviolet regulator in discretized light-cone quantization, Phys. Rev. D 58 (1998) 025005; O. Greenberg and S.S. Schweber, Clothed particle operators in simple models of quantum field theory, Nuovo Cimento 8 (1958) 378.

[10] S.J. Brodsky and S.D. Drell, The Anomalous Magnetic Moment And Limits On Fermion Substructure, Phys. Rev. D 22 (1980) 2236.

[11] S.S. Chabysheva and J.R. Hiller, An application of the light-front coupled-cluster method to the nonperturbative solution of QED, arXiv:1203.0250 [hep-ph].

[12] G.F. de Teramond and S.J. Brodsky, Light-front holography: A first approximation to QCD, Phys. Rev. Lett. 102 (2009) 081601. 